Tuberculose: desigualdade de renda e interação da Estratégia Saúde da Família e

\title{
Bolsa Família
}

\author{
Marcela Paschoal Popolin ${ }^{1}$, Maria Concebida da Cunha Garcia ${ }^{2}$, Luiz Henrique Arroyo ${ }^{3}$, \\ Mellina Yamamura ${ }^{4}$, Marcelino Santos Neto ${ }^{5}$, Ludmila Barbosa Bandeira Rodrigues ${ }^{6}$, \\ Ana Angélica Rêgo de Queiroz ${ }^{7}$, Inês Fronteira ${ }^{8}$, Ricardo Alexandre Arcêncio ${ }^{9}$
}

\footnotetext{
${ }^{1}$ Enfermeira, Doutora em Ciências. Ribeirão Preto, SP, Brasil. E-mail: marcelappopolin@gmail.com.

${ }^{2}$ Enfermeira, Doutora em Ciências. Ribeirão Preto, SP, Brasil. E-mail: concycg@yahoo.com.br.

${ }^{3}$ Fisioterapeuta, Mestre em Ciências. Discente do Programa de Pós-Graduação Interunidades de Doutoramento em Enfermagem da Escola de Enfermagem de Ribeirão Preto da Universidade de São Paulo, nível Doutorado. Ribeirão Preto, SP, Brasil. E-mail: luiz.arroyo@hotmail.com.

${ }^{4}$ Enfermeira, Doutora em Ciências. Discente do Programa de Pós-Graduação Interunidades de Doutoramento em Enfermagem da Escola de Enfermagem de Ribeirão Preto da Universidade de São Paulo, nível Pós-Doutorado. Ribeirão Preto, SP, Brasil. E-mail: mellinayamamura@yahoo.com.br. ${ }^{5}$ Farmacêutico, Doutor em Ciências. Professor Adjunto da Universidade Federal do Maranhão. Imperatriz, MA, Brasil. E-mail: marcelinosn@gmail.com.

${ }^{6}$ Enfermeira, Doutora em Ciências. Professora Adjunta da Universidade Federal de Mato Grosso. Sinop, MT, Brasil. E-mail: ludbbremerick@gmail.com. ${ }^{7}$ Enfermeira, Mestre em Enfermagem. Discente do Programa de Pós-Graduação Interunidades de Doutoramento em Enfermagem da Escola de Enfermagem de Ribeirão Preto da Universidade de São Paulo, nível Doutorado. Ribeirão Preto, SP, Brasil. E-mail: aninha_arego@hotmail.com.

${ }^{8}$ Enfermeira, Doutora em Saúde Internacional. Professora Auxiliar do Instituto de Higiene e Medicina Tropical da Universidade Nova de Lisboa. Lisboa, Portugal. E-mail: ifronteira@ihmt.unl.pt. ${ }^{9}$ Enfermeiro, Doutor em Enfermagem em Saúde Pública. Professor Associado da Escola de Enfermagem de Ribeirão Preto da Universidade de São Paulo. Ribeirão Preto, SP, Brasil. E-mail: ricardo@eerp.usp.br.
}

Recebido: 21/11/2016

Aceito: 01/06/2017.

Publicado: 04/09/2017.

Como citar esse artigo:

Popolin MP, Garcia MCC, Arroyo LH, Yamamura M, Santos Neto M, Rodrigues LBB et al. Preferência pelo tipo de parto, fatores associados à expectativa $e$ satisfação com o parto. Rev. Eletr. Enf. [Internet]. 2017 [acesso em: / _ ];19:a30. Disponível em: http://dx.doi.org/10.5216/ree.v19.44230.

\section{RESUMO}

Propôs-se verificar como a incidência e mortalidade por tuberculose (TB) se comportam em áreas cobertas pela Estratégia Saúde da Família e Bolsa Família, com ou sem desigualdade de renda. Estudo ecológico dos 26 Estados federados e o Distrito Federal do Brasil. Foram empregadas análises univariadas, multivariadas e análises espaciais. Na análise univariada, calculou-se a medida de posição (mediana) e a medida de dispersão (amplitude) das variáveis. $\mathrm{Na}$ análise multivariada, identificou-se sete grupos através da análise de agrupamento pelo método hierárquico. A construção dos mapas temáticos demonstrou uma distribuição heterogênea entre os grupos, devido às características particulares. O estudo evidenciou a realidade epidemiológica da TB no Brasil e seu comportamento em áreas cobertas pela Estratégia Saúde da Família e Programa Bolsa Família. Não observou diferenças no que se refere à incidência e mortalidade quando satisfatória cobertura desses programas, o que evidencia a relação da doença a outros determinantes.

Descritores: Renda; Programas Governamentais; Estratégia Saúde da Família; Tuberculose.

\section{INTRODUÇÃO}

Conhecida como uma das doenças infecciosas mais antigas do mundo, a tuberculose (TB) permanece como um problema de saúde pública sendo um dos agravos socialmente determinado pela pobreza, 
exclusão social, má distribuição de renda, ampla dispersão geográfica, epidemias de HIV e multidrogarresistência ${ }^{(1)}$.

O Brasil faz parte dos 30 países de alta carga priorizados pela Organização Mundial da Saúde (OMS) e ocupa a $20^{a}$ posição em número absoluto de casos, apresentando incidência de 41 casos por 100.000 habitantes e mortalidade de 2,7 óbitos por 100.000 habitantes $^{(2)}$.

Recentemente a OMS lançou a meta pelo Fim da TB (End TB), que tem como objetivos a eliminação da TB até 2050 (<1 caso por 100.000 habitantes) e redução da mortalidade em 95\% até 2035. Para tal, foram sugeridos três pilares que são sistemas universais e integrados de saúde (Pilar 1), proteção social (Pilar 2) e reforço às pesquisas (Pilar 3 ) para orientação das políticas e ações de controle. Em termos de estratégias nacionais que se aproximam destas premissas, tem-se a Estratégia Saúde da Família (ESF) que traz como filosofia a ampliação do acesso aos serviços de saúde, estando localizada estrategicamente nos territórios e o Programa Bolsa Família (PBF), que é um mecanismo de proteção social ${ }^{(2)}$.

Embora alguns estudos tenham mostrado o impacto de ambas as estratégias na redução da mortalidade infantil $^{(3)}$, no caso da TB, são poucos os estudos que têm evidenciado esse impacto na diminuição da incidência e mortalidade. Estudo realizado em Curitiba demonstrou que o aumento na cobertura da ESF repercutiu de forma positiva na redução do número de casos novos e na taxa de mortalidade relacionada à $\mathrm{TB}^{(4)}$. Autores $^{(5)}$ corroboram ao demonstrar que o Programa Bolsa Família conseguiu aumentar a taxa de adesão e cura dos pacientes de TB.

Ademais, outro estudo ${ }^{(6)}$ constatou o declínio da incidência da TB em países que tiveram maior Índice de Desenvolvimento Humano (IDH), menor mortalidade infantil e saneamento melhorado. Outros estudos sugerem que as estimativas de incidência da TB estão associadas a mudanças socioeconômicas e o estado geral de saúde da população(7).

Medidas econômicas adotadas no Brasil nos últimos anos frente à crise atual têm ameaçado constantemente a ESF e o PBF, o que torna premente evidenciar o quanto eles têm contribuído para melhoria dos indicadores epidemiológicos da TB. Por meio de uma revisão na literatura, não se observou estudos que demonstrassem o comportamento dos principais indicadores epidemiológicos da TB na presença dessas estratégias. Assim, diante dos objetivos assumidos de eliminação da TB e da potencialidade da ESF e PBF em promover melhoria das condições sociais e de saúde, é que se propõe verificar como a incidência e mortalidade por TB se comportam em áreas cobertas pela Estratégia Saúde da Família e Bolsa Família, com ou sem desigualdade de renda.

\section{MATERIAIS E MÉTODOS}

\section{Natureza do estudo}

Trata-se de um estudo ecológico que considerou os 26 Estados federados e o Distrito Federal do Brasil. 
Fonte de informações e coleta de dados

Os dados do estudo foram coletados em novembro de 2014 através dos sistemas de informação de domínio público do Departamento de informática do Sistema Único de Saúde do Brasil (DATASUS) e do Instituto Brasileiro de Geografia e Estatística (IBGE).

\section{Seleção das variáveis}

As variáveis consideradas para o estudo apresentam-se descritas no Quadro 1. Inicialmente, coletaram-se as taxas de incidência e mortalidade por TB do ano de 2012, os dados de cobertura da ESF e do PBF do ano de 2012 e, finalmente, o Índice de Gini do ano de 2003. Cabe destacar que os referidos anos correspondem àqueles disponíveis pelos sistemas de informação no período da coleta. Ademais, a escolha das variáveis se justifica por serem indicadores clássicos dos contextos da doença, dos serviços de saúde e social/condição de vida. 
Quadro 1: Variáveis utilizadas no estudo.

\begin{tabular}{|c|c|c|c|c|c|}
\hline Indicadores/Variáveis & Fórmula & Definições operacionais & Escores & Ano & Fonte \\
\hline \multirow{3}{*}{ Incidência da tuberculose } & \multirow{3}{*}{$\mathrm{IC}=\frac{\mathrm{n} \text { o de casos detectados determ. período de tempo }}{\text { total de indíviduos em risco no inicio do período }}$} & \multirow{3}{*}{$\begin{array}{l}\text { Refere-se ao número de novos casos que ocorrem em } \\
\text { uma população de indivíduos em risco durante um } \\
\text { determinado período de tempo. }\end{array}$} & Alta & \multirow{3}{*}{2012} & \multirow{3}{*}{$\begin{array}{l}\text { Sala de Apoio a } \\
\text { Gestão Estratégica } \\
\text { (SAGE) }\end{array}$} \\
\hline & & & Moderada & & \\
\hline & & & Baixa & & \\
\hline \multirow{3}{*}{$\begin{array}{l}\text { Mortalidade por tuberculose } \\
\text { respiratória }\end{array}$} & \multirow{3}{*}{$\mathrm{TMTB}=\frac{\mathrm{Dob}}{(\mathrm{Pi} \times 4)} \times 100,000$} & \multirow{3}{*}{$\begin{array}{l}\text { Tuberculose respiratória com confirmação ou não } \\
\text { histológica/bacteriológica (CID 10: A15.0 - A16.9) }\end{array}$} & Alta & \multirow{3}{*}{2012} & \multirow{3}{*}{$\begin{array}{c}\text { Sala de Apoio a } \\
\text { Gestão Estratégica } \\
\text { (SAGE) }\end{array}$} \\
\hline & & & Moderada & & \\
\hline & & & Baixa & & \\
\hline \multirow[b]{2}{*}{ Coeficiente de Gini } & \multirow{2}{*}{$\mathrm{G}=\frac{\alpha}{0,5}=2 \alpha$} & \multirow{2}{*}{$\begin{array}{l}\text { Utilizado para medir a desigualdade de distribuição de } \\
\text { renda e consiste entre } 0 \text { e } 1 \text {, em que } 0 \text { corresponde à } \\
\text { completa igualdade renda e } 1 \text { a completa desigualdade }\end{array}$} & Alto & \multirow[b]{2}{*}{2003} & \multirow{2}{*}{$\begin{array}{l}\text { Instituto Brasileiro } \\
\text { de Geografia e } \\
\text { Estatística (IBGE) }\end{array}$} \\
\hline & & & Baixo & & \\
\hline \multirow{3}{*}{$\begin{array}{l}\text { Cobertura Populacional da } \\
\text { Estratégia Saúde da Família } \\
\qquad \text { (ESF) }\end{array}$} & \multirow{3}{*}{$\mathrm{CPESF}=\frac{\mathrm{N} \cdot \mathrm{ESF} \times 3,450}{\mathrm{P} i} \times 100$} & \multirow{3}{*}{$\begin{array}{c}\text { Consiste em modelo de atenção à saúde pautado nas } \\
\text { ações de territorialização, da intersetorialidade, da } \\
\text { descentralização, da co- responsabilização e da } \\
\text { equidade. }\end{array}$} & Alta & \multirow{3}{*}{2012} & Sistema de \\
\hline & & & Média & & Informação da \\
\hline & & & Baixa & & $\begin{array}{l}\text { Atençăo Básica } \\
\qquad(\mathrm{SIAB})\end{array}$ \\
\hline \multirow{3}{*}{$\begin{array}{l}\text { Cobertura Populacional do } \\
\text { Programa Bolsa Família (PBF) }\end{array}$} & \multirow{3}{*}{$\mathrm{PBF}=\frac{\mathrm{N} \cdot \mathrm{BBF}}{\mathrm{P} i} \times 100$} & \multirow{3}{*}{$\begin{array}{c}\text { Programa de transferência direta de renda para as } \\
\text { famílias pobres e extremamente pobres. Ele é composto } \\
\text { por vários tipos de benefícios transferidos mensalmente } \\
\text { à população atendida. }\end{array}$} & Alta & \multirow{3}{*}{2012} & \multirow{3}{*}{$\begin{array}{l}\text { Secretaria de } \\
\text { Avaliação e Gestão } \\
\text { da Informação } \\
\text { (SAGI) }\end{array}$} \\
\hline & & & Média & & \\
\hline & & & Baixa & & \\
\hline
\end{tabular}

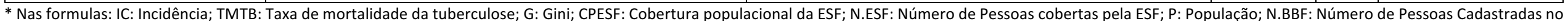
Programa Bolsa Família; CID 10: Classificação Internacional das Doenças. 


\section{Análise dos dados}

Realizou-se a análise descritiva, calculando-se a medida de posição (mediana) e a medida de dispersão (amplitude) das variáveis em estudo por meio do software Statistica 7.0. Consecutivamente, os Estados foram ordenados de forma decrescente e divididos em quartis, resultando em três níveis para taxas de incidência e mortalidade por TB (altas taxas, taxas moderadas e baixas taxas de incidência e mortalidade por TB); em dois níveis para o Índice de Gini (alta e baixa desigualdade de renda) e em três níveis para a cobertura de ESF e PBF (alta, média e baixa cobertura de ESF e PBF).

Aplicou-se ainda a análise multivariada, método estatístico que analisa simultaneamente múltiplas medidas sobre cada observação em investigação. Para isso, as variáveis foram padronizadas através do método de transformação $Z$, em que a matriz de correlação passou a ser exatamente igual à matriz de covariância ${ }^{(8)}$. Depois, efetuou-se a análise de agrupamento por nível hierárquico com o processamento da distância euclidiana e o método de Ward. Essa técnica deu origem ao dendograma (Figura 1) e permitiu de acordo com as variáveis investigadas a junção de grupos "semelhantes". Através do dendrograma é possível verificar a similaridade entre as observações, considerando que a menor distância entre elas corresponde ao maior nível de semelhança ${ }^{(8)}$.

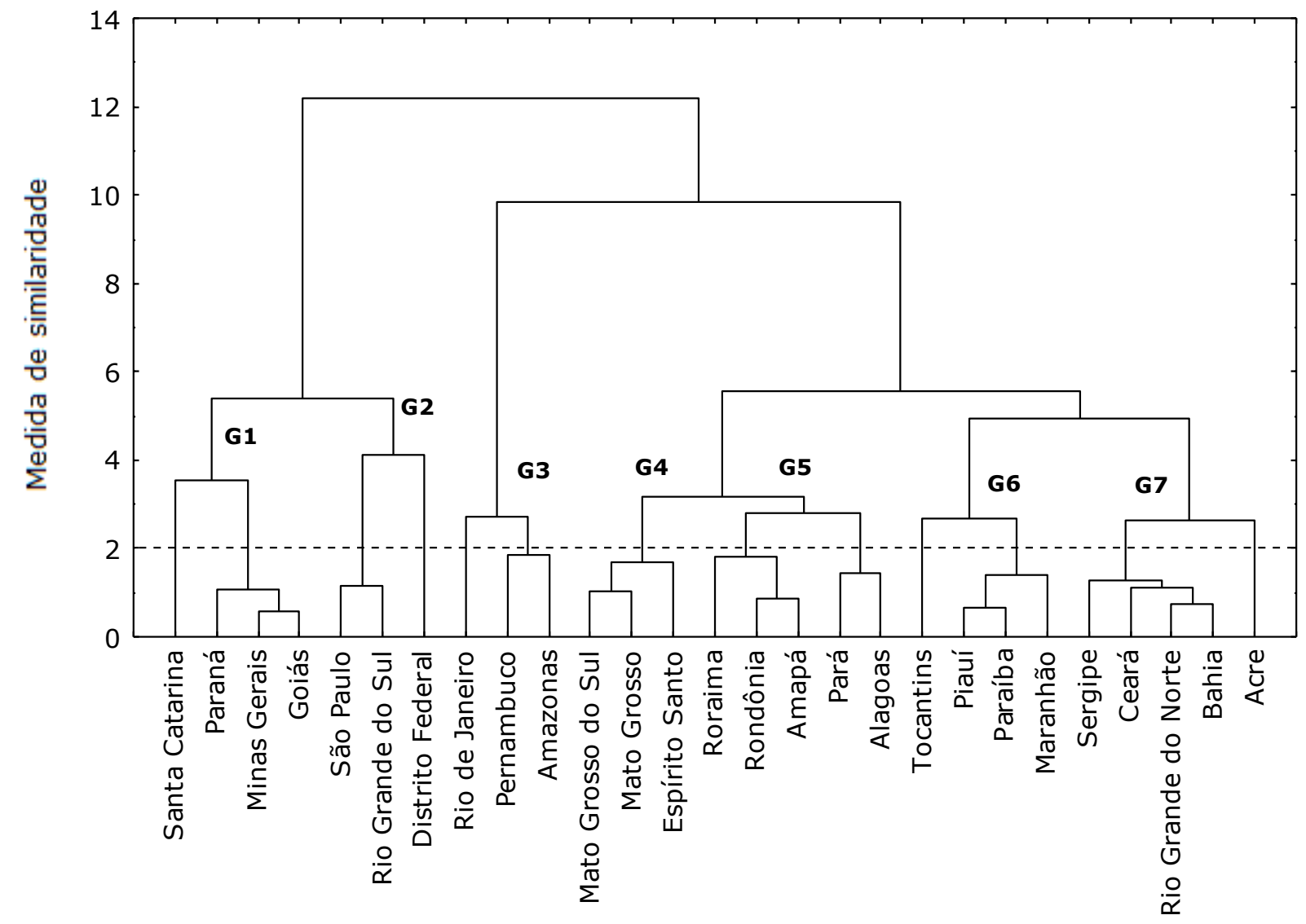

Figura 1: Dendrograma obtido da aplicação das Análises de Agrupamento por níveis hierárquicos, Brasil, (2003-2012).

Para facilitar a visualização dos dados agrupados foram construídos mapas temáticos utilizando 
o Software ArcGis 4.2.2. a fim de verificar a distribuição espacial das variáveis analisadas considerando as características similares dos municípios (agrupados).

\section{Aspectos éticos}

De acordo com recomendações da Comissão Nacional de Ética em Pesquisa (CONEP), por se tratarem de dados secundários, de domínio público em que nenhum participante da pesquisa foi identificado, dispensa-se a apresentação deste ao Comitê de Ética em Pesquisa (CEP).

\section{RESULTADOS}

A Figura 1 apresenta o dendograma resultante da análise de agrupamento pelo método hierárquico, que consiste numa estrutura contendo sete grupos. A análise por método não hierárquico (k-médias), processada com a formação desses grupos, permitiu caracterizar o Grupo 1 com quatro Estados (Santa Catarina - SC; Paraná - PR; Minas Gerais - MG e Goiás - GO), o Grupo 2 com três Estados (Rio Grande do Sul - RS; São Paulo - SP e Distrito Federal - DF), o Grupo 3 com três (Rio de Janeiro - RJ; Pernambuco - PE e Amazonas - AM), o Grupo 4 com três Estados (Mato Grosso - MT; Mato Grosso do Sul - MS e Espírito Santo - ES), o Grupo 5 com cinco Estados (Pará - PA; Rondônia RO; Alagoas - AL; Amapá - AP e Roraima - RR), o Grupo 6 com quatro (Paraíba - PB; Maranhão (MA); Piauí - PI e Tocantins - TO) e o Grupo 7 com cinco Estados (Acre - AC; Ceará - CE; Bahia - BA, Rio Grande do Norte - RN e Sergipe - SE).

A Tabela 1 apresenta os dados resultantes da estatística descritiva por Estados e Distrito Federal de acordo com os grupos formados na análise de agrupamento. 
Tabela 1: Dados das variáveis de acordo com os Estados brasileiros e Distrito Federal, Brasil, (2003-2012).

\begin{tabular}{|c|c|c|c|c|c|c|c|c|c|c|c|}
\hline Estado ou DF & Grupos & $\begin{array}{c}\text { Taxa de } \\
\text { incidência da } \\
\text { tuberculose }\end{array}$ & Mediana & $\begin{array}{c}\text { Taxa de } \\
\text { mortalidade } \\
\text { por tuberculose }\end{array}$ & Mediana & $\begin{array}{l}\text { Índice } \\
\text { de Gini }\end{array}$ & Mediana & $\begin{array}{l}\text { Cobertura da } \\
\text { Estratégia } \\
\text { Saúde da } \\
\text { Família (ESF) }\end{array}$ & Mediana & $\begin{array}{c}\text { Cobertura } \\
\text { populacional do } \\
\text { Programa Bolsa } \\
\text { Família (PBF) }\end{array}$ & Mediana \\
\hline Santa Catarina (SC) & 1 & 28,40 & \multirow{4}{*}{19,25} & 0,68 & \multirow{4}{*}{1,08} & 0,49 & \multirow{4}{*}{0,46} & 72,50 & \multirow{3}{*}{69,85} & 8,68 & \multirow{4}{*}{17,76} \\
\hline Paraná (PR) & 1 & 20,35 & & 0,93 & & 0,47 & & 61,70 & & 15,55 & \\
\hline Minas Gerais (MG) & 1 & 18,14 & & 1,37 & & 0,46 & & 74,14 & & 22,30 & \\
\hline Goiás (GO) & 1 & 15,01 & & 1,23 & & 0,45 & & 67,20 & & 19,96 & \\
\hline Rio Grande do Sul (RS) & 2 & 43,35 & \multirow{3}{*}{38,57} & 2,13 & \multirow{3}{*}{1,99} & 0,48 & \multirow{3}{*}{0,48} & 42,94 & & 14,91 & \multirow{3}{*}{11,50} \\
\hline São Paulo (SP) & 2 & 38,57 & & 1,99 & & 0,45 & & 36,85 & 36,85 & 10,68 & \\
\hline Distrito Federal (DF) & 2 & 13,44 & & 0,49 & & 0,52 & & 18,43 & & 11,50 & \\
\hline Amazonas (AM) & 3 & 65,72 & \multirow{3}{*}{65,60} & 3,45 & \multirow{3}{*}{4,14} & 0,50 & \multirow{3}{*}{0,50} & 64,39 & & 37,23 & \multirow{3}{*}{37,23} \\
\hline Rio de Janeiro (RJ) & 3 & 65,60 & & 4,56 & & 0,50 & & 49,63 & 64,39 & 16,21 & \\
\hline Pernambuco (PE) & 3 & 48,65 & & 4,14 & & 0,50 & & 86,39 & & 42,75 & \\
\hline Mato Grosso (MT) & 4 & 41,66 & \multirow{3}{*}{36,12} & 2,63 & \multirow{3}{*}{2,51} & 0,47 & \multirow{3}{*}{0,47} & 74,91 & \multirow{3}{*}{72,59} & 20,93 & \multirow{3}{*}{20,16} \\
\hline Mato Grosso do Sul (MS) & 4 & 36,12 & & 2,51 & & 0,46 & & 92,75 & & 20,07 & \\
\hline Espírito Santo (ES) & 4 & 34,57 & & 2,06 & & 0,50 & & 70,26 & & 20,16 & \\
\hline Pará (PA) & 5 & 43,41 & \multirow{5}{*}{32,91} & 2,71 & \multirow{5}{*}{2,00} & 0,44 & \multirow{5}{*}{0,45} & 79,64 & \multirow{5}{*}{79,64} & 39,85 & \multirow{5}{*}{35,21} \\
\hline Rondônia (RO) & 5 & 34,46 & & 1,38 & & 0,46 & & 83,40 & & 26,99 & \\
\hline Alagoas (AL) & 5 & 32,91 & & 3,03 & & 0,47 & & 77,14 & & 48,57 & \\
\hline Amapá (AP) & 5 & 29,20 & & 2,00 & & 0,45 & & 81,88 & & 30,57 & \\
\hline Roraima (RR) & 5 & 25,77 & & 1,49 & & 0,44 & & 59,24 & & 37,80 & \\
\hline Paraíba (PB) & 6 & 28,96 & \multirow{4}{*}{24,77} & 1,93 & \multirow{4}{*}{1,87} & 0,46 & \multirow{4}{*}{0,46} & 98,16 & \multirow{4}{*}{97,98} & 45,42 & \\
\hline Maranhão (MA) & 6 & 27,71 & & 2,12 & & 0,43 & & 88,88 & & 51,83 & \multirow{3}{*}{47,86} \\
\hline Piauí (PI) & 6 & 21,83 & & 1,80 & & 0,46 & & 98,81 & & 50,30 & \\
\hline Tocantins (TO) & 6 & 12,97 & & 0,56 & & 0,47 & & 97,80 & & 36,30 & \\
\hline Acre $(A C)$ & 7 & 43,33 & \multirow{5}{*}{33,77} & 1,05 & & 0,53 & & 97,04 & & 40,64 & \\
\hline Ceará (CE) & 7 & 38,28 & & 2,53 & & 0,51 & & 83,24 & & 46,33 & \\
\hline Bahia (BA) & 7 & 33,77 & & 2,41 & 2,07 & 0,49 & 0,50 & 83,50 & 83,50 & 43,82 & 42,87 \\
\hline Rio Grande do Norte (RN) & 7 & 27,75 & & 2,07 & & 0,49 & & 77,42 & & 38,67 & \\
\hline Sergipe (SE) & 7 & 24,30 & & 1,89 & & 0,50 & & 94,44 & & 42,87 & \\
\hline
\end{tabular}


No Quadro 2 está descrita a classificação dos grupos de acordo com os escores de cada variável. Para a taxa de incidência e a taxa de mortalidade o escore mais prevalente foi aquele com taxas moderadas, respectivamente $(24,30-41,65) ;(1,30-2,51)$; para o Índice de Gini o escore mais predominante foi baixo Índice $(\leq 0,47)$ e para a cobertura da ESF e PBF o mais influente foi baixa cobertura, respectivamente $(<59,24)$; $(<19,96)$.

Quadro 2: Classificação dos grupos segundo escores, Brasil, 2016.

\begin{tabular}{|c|c|c|c|c|c|}
\hline Grupos & Taxa de incidência & Taxa de mortalidade & Índice de Gini & Cobertura ESF & Cobertura PBF \\
\hline Grupo 1 & Baixa & Baixa & Baixo & Baixa & Baixa \\
\hline Grupo 2 & Moderada & Moderada & Alto & Baixa & Baixa \\
\hline Grupo 3 & Alta & Alta & Alto & Baixa & Baixa \\
\hline Grupo 4 & Moderada & Alta & Baixo & Baixa & Média \\
\hline Grupo 5 & Moderada & Moderada & Baixo & Média & Baixa \\
\hline Grupo 6 & Baixa & Baixa & Baixo & Alta & Alta \\
\hline Grupo 7 & Moderada & Moderada & Alto & Alta & Alta \\
\hline
\end{tabular}

A Figura 2 por sua vez, apresenta a distribuição desses grupos segundo Estados brasileiros e Distrito Federal. Os Estados pertencentes ao seu respectivo grupo estão representados por uma cor diferente.

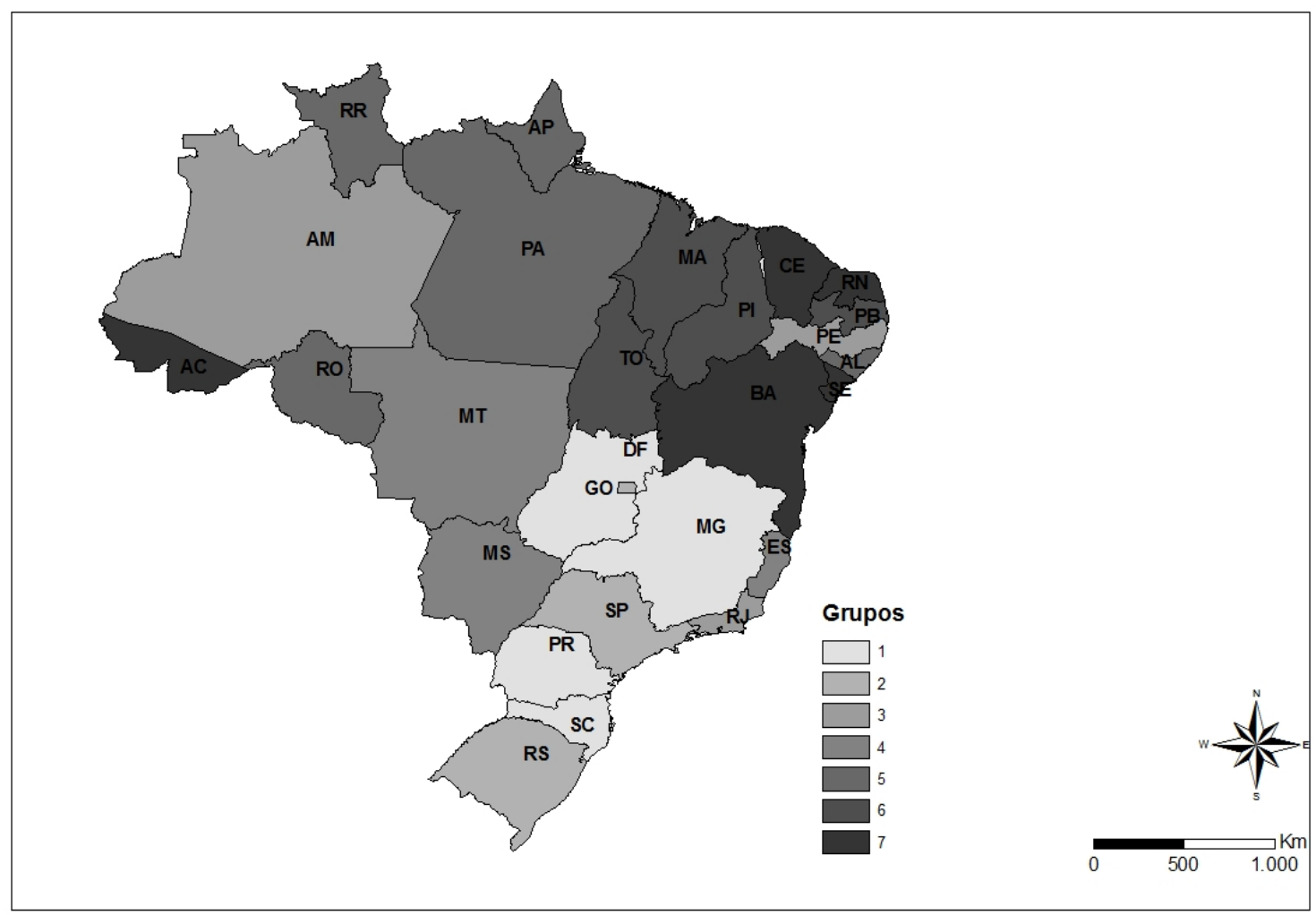

Figura 2: Mapa temático dos grupos formado pelos Estados brasileiros e Distrito Federal, Brasil, 2016.

\section{DISCUSSÃO}

Propôs-se verificar como a incidência e mortalidade por TB se comportam em áreas cobertas pela Estratégia Saúde da Família e Bolsa Família, com ou sem desigualdade de renda.

Observou-se que as altas taxas de incidência e mortalidade por TB ocorreram de forma heterogênea entre os estados e as macrorregiões brasileiras. Verificou-se ainda, que esta situação epidemiológica ocorreu 
tanto em áreas com alta ou baixa cobertura de ESF e do PBF, podendo haver outras questões relacionadas ao problema da TB, que não somente essas estratégias.

É importante destacar que, embora o Brasil tenha um nível satisfatório de deteç̧ão de casos de TB (acima de $70 \%$ ), o problema está em garantir que o paciente conclua o seu tratamento (bem abaixo dos $85 \%$ recomendado pela OMS) e ao diagnóstico tardio ${ }^{(9)}$ que pode ser favorável a elevada taxa de mortalidade em alguns Estados.

Outra situação evidenciada no estudo foram áreas com alta cobertura de ESF e PBF e resultados pífios em relação à incidência de TB, que pode ser em decorrência de fragilidades notadamente da ESF (Grupo 6) na detecção de casos, possivelmente pela ocorrência de subnotificação, considerando a situação social nessas localidades e a própria extensão territorial.

O estudo demonstrou ainda que o Grupo 1 se compôs em sua maioria de Estados da região Sul e Sudeste, que trazem melhores indicadores em termos de distribuição de renda (Gini mais baixo em relação aos demais) e baixa a cobertura de ESF e Bolsa Família. Embora a ESF tenha sido pensada para todos (ideário de Alma Ata), no Brasil tem se vigorado a ideia de uma Atenção Primária à Saúde (APS) seletiva, alocando a mesma em regiões mais carentes e pobres, semelhantemente aos resultados do estudo ${ }^{(10)}$.

Os resultados mostraram também uma relação entre nível de distribuição de renda e Programa Bolsa Família, verificando que os Estados do Norte e Nordeste apresentaram Gini mais elevado e por sua vez, maior cobertura de benefício social. Proporção essa bem menor nos Estados do Sul e Sudeste, que tradicionalmente se apresentam com maior Produto Interno Bruto e melhor distribuição de renda quando comparado aos outros Estados.

A desigualdade de renda é um problema patente no Brasil, destacando-se como um dos países líderes em desigualdade perdendo para países como África do Sul, Serra Leoa e Lesoto ${ }^{(11)}$, e exerce um efeito portentoso na cadeia de progressão da TB e se há interesse pela sua eliminação devem ser direcionados aos seus determinantes.

Nessa conjuntura, historicamente, a TB é associada a altos índices de pobreza e baixo desenvolvimento socioeconômico. O seu paradigma epidemiológico parte do pressuposto de que a doença se distribui de maneira desigual nos territórios e essa desigualdade se reflete na condição de vida. Alguns autores relatam que, onde há privação social, particularmente em áreas de habitação pobre, desemprego elevado e baixa renda, deve haver um incentivo para as ações de busca de sintomáticos respiratórios e diagnóstico precoce da TB, pois o agravamento das condições sociais e econômicas resulta em degradação significativa das condições de vida, aumentando a vulnerabilidade e, consequentemente, o risco de adoecer pela doença ${ }^{(1)}$.

Assim, reforça-se a necessidade do controle da TB englobar a correção dessas desigualdades considerando o impacto das condições socioeconômicas sobre o agravamento da situação epidemiológica da doença. Para tal, ações relativas aos serviços de saúde devem ser voltadas para a promoção da saúde e prevenção das doenças facilitando o acesso ao diagnóstico e tratamento, aumentando os esforços globais e locais na perspectiva de criação e efetivação de políticas públicas que atuem nos riscos que contribuem para 
o adoecimento por TB.

Alguns autores afirmam que a inclusão do tratamento de problemas de saúde como a TB nas condicionalidades do PBF poderia ser uma iniciativa governamental para reduzir o impacto da pobreza na saúde dessa população ${ }^{(12)}$. Especialmente em relação a TB, essa medida poderá contribuir para a adesão ao tratamento, principalmente porque complementaria a dieta alimentar do paciente e ajudaria a combater os efeitos colaterais do medicamento e da falta de alimentação.

Autores $^{(13)}$ apontam que estudos focando a ESF poderiam contribuir com a gestão em saúde, a identificação de problemas, a reorientação das ações e a incorporação de novas práticas sanitárias, como as relativas às áreas estratégicas da APS, dentre elas o controle da TB.

A perspectiva para tais programas é que estes serviriam de porta de entrada para os serviços de saúde, promovendo o acesso a uma assistência básica de boa qualidade e, principalmente, o controle da doença. Autores apontam que existe uma associação inversa entre o acesso aos serviços de saúde e a TB, notadamente nas taxas de incidência e mortalidade pela doença ${ }^{(14)}$, visto que a ESF, pelas suas características de integralidade, oportunidade, continuidade da atenção e manejo dos problemas de saúde mais prevalentes $^{(13)}$, poderá contribuir para evitar ou diminuir a ocorrência da TB bem como sua evolução.

Contudo segundo estudos, na prática, o processo de intersetorialidade das ações e estratégias sociais, econômicas e de saúde é recente e sofre inúmeras fragilidades processuais que dificultam o atendimento às famílias. Um dos fatores dificultadores desse sistema refere-se à verticalização dos programas governamentais, onde a hegemonia na tomada de decisão enfraquece as ações realizadas em níveis mais micro do sistema ${ }^{(15)}$.

Vê-se ante esses resultados, uma hipótese da capacidade dos municípios em cumprir e acompanhar tais situações ou determinantes, como levantada por autores ${ }^{(16)}$. Diante disso, o Estado buscou incentivar financeiramente os municípios que obtivessem determinados níveis de qualidade de gestão do PBF. No entanto, essas estratégias sofrem diversas críticas, pela centralidade do incentivo no financiamento ${ }^{(16)}$.

Outro aspecto a ser trabalhado refere-se aos gestores ou profissionais da saúde que desconhecem os arranjos e organização do PBF, causando a inviabilidade do atendimento de forma plena pelos programas sociais. Ademais, a alegação dos profissionais sobre o nível de articulação desses programas dificulta o vínculo junto às famílias atendidas, que por sua vez, temem o corte do benefício perante as visitas de rotina da equipe de saúde ${ }^{(17)}$.

Embora a atuação dessas estratégias possa produzir um efeito positivo, os resultados ainda são questionáveis ou pífios, comprometendo o delineamento de políticas públicas com impacto negativo nos indicadores de morbimortalidade da TB no país ${ }^{(17)}$. Por outro lado, o estudo permitiu mostrar um panorama epidemiológico da TB no país e ainda refletir sobre a cobertura da ESF e PBF e a potencialidade dessas estratégias no tocante à eliminação da TB. Embora tenham sido apresentadas situações muito diversas entre os cenários investigados, não podendo concluir se tais estratégias contribuem para a melhoria dos indicadores epidemiológicos, mas tem apontando para progressos nas condições sociais de família, retirando 
muitos da pobreza extrema. A ESF potencializada pelo PBF presta um grande serviço à sociedade brasileira e, portanto, deve haver maior investimento como política de Estado.

Destaca-se ainda a relevância do estudo ao utilizar a abordagem geoecológica para classificar os Estados em relação aos indicadores epidemiológicos da TB. Esses resultados podem subsidiar os gestores e profissionais de saúde no planejamento, monitoramento e avaliação das suas ações em saúde e quanto falta para alcançar a meta de eliminação da doença. Além disso, ressalta-se a importância dessa abordagem no papel do enfermeiro na qual poderá coletar as informações e executar projetos de intervenção sanitária, construir mapas e discuti-los com os diversos atores do seu território, tais como usuários, profissionais de saúde, gestores e estudantes.

Ademais, estudos que consideram a análise espacial podem constituir uma ferramenta muito apropriada para a avaliação dos sistemas e serviços de saúde, haja vista que os mapas podem sensibilizar mais gestores e trabalhadores, propiciando diagnóstico situacional da realidade, levantamento de hipóteses relacionadas ao problema e compreensão da sua complexidade e determinantes ${ }^{(18)}$.

Dentre as limitações do estudo, destaca-se a heterogeneidade dos sistemas de saúde na produção dos seus dados, registro dos dados, cobertura dos dados, que pode variar no tempo, nas regiões e as potenciais perdas de informações. Outra limitação refere-se ao fato de ter utilizado os Estados federados e não os municípios como unidade de análise, sendo o primeiro muito heterogêneo com grande diversidade em termos dos seus indicadores epidemiológicos, sociais e a própria cultura, embora haja uma normativa federal para alimentar esses indicadores.

Considerando as dimensões territoriais do Brasil, quase que um continente, trabalhar suas menores unidades geográficas como setores censitários, é um grande desafio, todavia o estudo avança no conhecimento ao revelar uma realidade situacional da TB no país e o comportamento da doença especialmente por meio de seus dois principais indicadores (incidência e mortalidade) ante a ESF e PBF, que são duas principais estratégias a serem utilizadas para eliminação da TB.

\section{CONCLUSÕES}

Os resultados do estudo apontaram que independente da cobertura de ESF e PBF, as taxas de incidência e mortalidade por TB oscilaram, tendo áreas com alta cobertura de ESF e PBF e com detecção de casos alta ou baixa. Assim, não se pode inferir que áreas com maior cobertura dessas estratégias têm também melhores indicadores epidemiológicos, premissa inicial do estudo. Os Estados do Sul e Sudeste foram os que apresentaram menor desigualdade social (Gini) e baixa cobertura da ESF do PBF. Os Estados das regiões Norte e Nordeste apresentaram maior cobertura da ESF e PBF.

O conhecimento da situação epidemiológica nos estados brasileiros no tocante a incidência e óbitos por TB e a sua relação com a ESF e o PBF e a desigualdade social, suscitou reflexões quanto aos caminhos a serem trilhados no país para a eliminação da TB. A estratégia End TB sugeriu dois pilares inovadores para o alcance da meta de eliminação, o primeiro se refere à universalidade e a integração dos seus sistemas para 
acessibilidade do paciente de TB, um processo que já vem avançando no Brasil com a Constituição de 1988 e pelo reforço a política da APS por meio da ESF. Outro pilar é a proteção social, em que o PBF cumpre esse papel e, portanto, pode contribuir para retirar pessoas da pobreza extrema, o que muito provavelmente traz impacto positivo a TB. Todavia, alguns aspectos ficaram lacunares talvez porque a dinâmica da doença se imbrica a outras variáveis não captadas no estudo, como as rotinas dos serviços, a cultura da população, as micropolíticas intra-estados, formação dos profissionais, entre outros, o que deve ser avançado em estudos futuros.

\section{Agradecimentos}

Fundação de Amparo à Pesquisa do Estado de São Paulo (FAPESP), processo no 2013/22486-2.

\section{REFERÊNCIAS}

1. San Pedro A, Oliveira RM. Tuberculose e indicadores socioeconômicos: revisão sistemática da literatura. Rev Panam Salud Pública [Internet]. 2013 [acesso em: 04 set. 2017];33(4):294-301. Disponível em:

http://dx.doi.org/10.1590/S1020-49892013000400009.

2. World Health Organization. Global Tuberculosis Report 2015 [Internet]. Geneva: World Health Organization; 2016 [acesso em: 04 set. 2017]. Disponível em:

http://apps.who.int/iris/bitstream/10665/191102/1/9789241565059_eng.pdf.

3. Rasella D, Aquino R, Santos CA, Paes-Sousa R, Barreto ML. Effect of a conditional cash transfer programme on childhood mortality: a nationwide analysis of Brazilian municipalities. Lancet [Internet]. 2013 [acesso em: 04 set. 2017];382(9886):57-64. Disponível em: http://dx.doi.org/10.1016/S0140-6736(13)60715-1.

4. Santos CRB, Magalhães R. Pobreza e Política Social: a implementação de programas complementares do Programa Bolsa Família. Cien Saude Colet [Internet]. 2012 [acesso em: 04 set. 2017];17(5):1215-24. Disponível em:

http://dx.doi.org/10.1590/S1413-81232012000500015.

5. Torrens AW, Rasella D, Boccia D, Maciel EL, Nery JS, Olson ZD et al. Effectiveness of a conditional cash transfer programme on TB cure rate: a retrospective cohort study in Brazil. Trans R Soc Trop Med Hyg [Internet]. 2016 [acesso em: 04 set. 2017];110(3):199-206. Disponível em: http://dx.doi.org/10.1093/trstmh/trw011.

6. Arinaminpathy $\mathrm{N}$, Dye $\mathrm{C}$. Health in financial crises: economic recession and tuberculosis in Central and Eastern Europe. J R Soc Interface [Internet]. 2010 [acesso em: 04 set. 2017];7(52):1559-69. Disponível em:

http://dx.doi.org/10.1098/rsif.2010.0072.

7. Oxlade O, Schwartzman K, Behr MA, Benedetti A, Pai M, Heymann J et al. Global tuberculosis trends: a reflection of changes in tuberculosis control or in population health? Int J Tuberc Lung Dis [Internet]. 2009 [acesso em: 04 set. 2017];13(10):1238-46. Disponível em:

http://www.ingentaconnect.com/contentone/iuatld/ijtld/2009/00000013/00000010/art00010.

8. Mingoti SA. Análise de dados através de métodos de estatística multivariada - uma abordagem aplicada. Belo Horizonte: UFMG; 2005. 297 p.

9. Villa TC, Ruffino-Netto A, Scatena LM, Andrade RL, Brunello ME, Nogueira JA et al. Health services performance for TB treatment in Brazil: a cross-sectional study. BMC Health Serv Res [Internet]. 2011 [acesso em: 04 set. 2017];11(1):241. Disponível em: https://doi.org/10.1186/1472-6963-11-241.

10. Giovanella L. Atenção Primária à Saúde seletiva ou abrangente? Cad Saude Publica [Internet]. 2008 [acesso em: 04 set. 2017];24(supl. 1):s21-3. Disponível em: http://dx.doi.org/10.1590/s0102-311X2008001300005.

11. OECD. Active with Brazil [Internet]. Paris: OECD; 2015 [acesso em: 04 set. 2017]. Disponível em: http://www.oecd.org/brazil/Brazil\%20brochureWEB.pdf.

12. Pinheiro RS, Oliveira GP, Oliveira EXG, Melo ECP, Coeli CM, Carvalho MS. Determinantes sociais e autorrelato de tuberculose nas regiões metropolitanas conforme a Pesquisa Nacional por Amostra de Domicílios, Brasil. Rev Panam 
Salud Pública [Internet]. 2013 [acesso em: 04 set. 2017];34(6):446-51. Disponível em:

http://www.scielosp.org/scielo.php?script=sci_arttext\&pid=S1020-49892013001200011\&lng=en\&nrm=iso\&tlng=pt.

13. Backes DS, Backes MS, Erdmann AL, Büscher A. O papel profissional do enfermeiro no Sistema Único de Saúde: da saúde comunitária à estratégia de saúde da família. Cien Saude Colet [Internet]. 2012 [acesso em: 04 set.

2017];17(1):223-30. Disponível em: http://dx.doi.org/10.1590/S1413-81232012000100024.

14. Kronman AC, Ash AS, Freund KM, Hanchate A, Emanuel EJ. Can primary care visits reduce hospital utilization among Medicare beneficiaries at the end of life? J Gen Intern Med [Internet]. 2008 [acesso em: 04 set.

2017];23(9):1330-5. Disponível em: http://dx.doi.org/10.1007/s11606-008-0638-5.

15. Marques FC, Ribeiro KSMA, Santos WQ. Intersetorialidade: possibilidade de parcerias entre a Estratégia Saúde da Família e a Pastoral da Criança. Saúde em Debate [Internet]. 2012 [acesso em: 04 set. 2017];36(95):544-53. Disponível em: http://dx.doi.org/10.1590/S0103-11042012000400006.

16. Monteiro DAA, Ferreira MAM, Teixeira KMD. Determinantes da gestão do Programa Bolsa Família: análise do índice de gestão descentralizada em Minas Gerais. Saúde e Soc [Internet]. 2009 [acesso em: 04 set. 2017];18(2):21426. Disponível em: http://dx.doi.org/10.1590/S0104-12902009000200005.

17. Integração entre o Bolsa Família e o Programa de Saúde da Família: desafios estratégicos [Internet]. Brasília (DF): UNDP/IPC-IG; c2017 [acesso em: 04 set. 2017]. Disponível em: http://www.ipcig.org/publication/mds/17P.pdf.

18. Arcêncio RA. Tecnologias em saúde para análise espacial e diagnóstico situacional dos territórios: contribuições para a enfermagem. Rev Bras Enferm [Internet]. 2015 [acesso em: 04 set. 2017];68(6):999-1000. Disponível em: http://dx.doi.org/10.1590/0034-7167.2015680601i. 